Europe's Journal of Psychology, 7(4), pp. 597-603

www.ejop.org

\title{
On curiosity and passion for science: Interview with Emily Holmes
}

\section{By Vlad Glăveanu EJOP Editor}

In this interview Prof. Emily Holmes addresses some of the most recent developments in the area of experimental psychopathology. At the core of her interest for this field stands a pervasive preoccupation for unpacking the importance of the imaginary in terms of our mental functioning and, in particular, the relationship between the mental imagery and emotion. Prof. Holmes explores, in this context, several important issues for academics and practitioners alike, from methodological aspects to the broader concern for making research results directly applicable and disseminating them effectively to different groups and audiences. In this respect much can be learned from Prof. Holmes's career trajectory and, towards the end, she offers students and early career researchers some valuable advice about the importance of curiosity and passion for science in succeeding as a researcher, as a psychologist and, overall, as a successful "academic citizen".

Emily A. Holmes is a Professor in Clinical Psychology and Wellcome Trust Clinical Fellow at the University of Oxford's Department of Psychiatry. She set up the EPACT Research Group with support from the Royal Society, the ESRC, MRC and the John Fell OUP Research Fund. Emily is a Clinical Psychologist with a PhD in Cognitive Neuroscience. Her overarching research interest is investigating mental imagery and emotion in psychopathology for cognitive therapy. She has published extensively in this field as well as related areas (e.g. processing biases, the role of flashbacks in posttraumatic stress disorder, depression, bipolar and anxiety disorders, etc.). Prof. Holmes is also the recipient of several prestigious awards such as the BPS May Davidson Award (2007) and Spearman Medal (2010), and the EFPA Comenius Award (2011).

Address for correspondence: Prof Emily Holmes, Department of Psychiatry, Oxford University, Warneford Hospital, Oxford, OX3 7JX, UK.

Email: emily.holmes@psych.ox.ac.uk 
EJOP: Prof. Holmes, you work in the past years had made significant contributions to our understanding of trauma memory, emotion, mental imagery, depression and bipolar disorder, spanning the fields of clinical, cognitive and experimental psychology. What would you say are the common threads within your research and how can insights from different disciplines come together to further our understanding of psychological disorders?

Prof. Holmes: The common thread in my work is a fascination with mental imagery how we can 'see' things in our mind's eye (or mind's ear, nose etc.) in the absence of direct perception. In psychology, we still have so much to find out about mental imagery compared to say verbal thought or verbal memory. So this makes it an exciting frontier to explore. If we saw it like a spider's web, we would have lots of different threads leading to different areas to which imagery is relevant, and the spider in the middle of the web is a driver - an interest in translational questions i.e. how does mental imagery relate to something we want to know about psychopathology? It might be the relationship between mental imagery and emotion, it might be mental imagery and behaviour and it also might be mental imagery across the different disorders. This means we can pose lots of exciting questions along these imagery threads which explore and bridge different disciplines!

I think it's very exciting working across disciplines. When we talk about disciplines, we are talking about two different kinds; disciplines within psychology and crossdisciplinary work in the broader sense. Making links and collaborations on both levels can be extremely productive and worthwhile. There are lots of different disciplines within psychology which our experimental psychopathology research draws on and speaks to, for example clinical psychology (e.g. cognitive behavioural therapy Hackmann, Bennett-Levy, \& Holmes, 2011) as well as say social psychology (e.g. prejudice), cognitive psychology (e.g. memory), experimental psychology, individual differences etc. We also work at times with neuroscience methods e.g. fMRI brain imaging. In other work we have researched across disciplines from psychology to medicine and also as varied as philosophy (Holmes, Sandberg, \& lyadurai, 2010), law (Holmes, 2008) and mathematics. For example, we have recently been working with a mathematical biologist on mood which has opened up some fascinating avenues for future research (Bonsall, Wallace-Hadrill, Geddes, Goodwin, \& Holmes, 2011). We enjoy involving artists in discussion about our work and they have even helped us make stimuli (Pictet, Coughtrey, Mathews, \& Holmes, 2011)

EJOP: Your doctoral work at the University of Cambridge, completed in 2005, explored the relationship between mental imagery and emotion. Can you please 
describe briefly your findings and how you have built up on them in the years that followed?

Prof. Holmes: My work with Andrew Mathews tested the hypothesis that mental imagery has a special relationship with emotion. We found evidence to support this hypothesis as our work showed that mental imagery has a greater impact on emotion compared to verbal thoughts (for a review see Holmes \& Mathews, 2010). This work has been a driver that has fuelled much of my work since, as we've really tried to put mental imagery at the centre as a focus for mental health treatment targets. We've started to develop treatment programmes of research, taking the link between mental imagery and emotion into bipolar disorder, into unipolar depression and into PTSD and flashbacks. The treatment development work we do is underpinned with a strong interest in cognitive neuroscience.

EJOP: One of your earlier research projects, at UCL, was related to the role of flashbacks in posttraumatic stress disorder (PTSD). Research into PTSD has been developing greatly and it constitutes now a very vibrant field with numerous practical implications. What do you think we have learned about PTSD in recent years and what remains to be studied in the near future?

Prof. Holmes: We have excellent treatments for full-blown PTSD and these are now recommended by the UK guidelines for evidence-based treatment (National Institute for Health and Clinical Excellence, 2005). In this case, the treatment recommended is trauma-focussed cognitive behavioural therapy (CBT). So once someone has established, full-blown PTSD, those treatments are very good. However, we lack evidence-based treatments to offer people in the immediate aftermath of trauma, for example if there is a bomb or a natural disaster happens, what can we recommend can be done then? So what we're focussing our research on is the idea of preventing at a very early stage the build-up of flashbacks (distressing imagery-based memories of the trauma). Flashback are the hallmarks of PTSD and we have shown in the lab that we can dampen them down by playing computer games such as Tetris (Holmes, James, Kilford, \& Deeprose, 2010).

EJOP: In more recent work you have focused on depression and bipolar disorders. What are your current (and future) research projects?

Prof. Holmes: We're trying slowly but surely to look at ways of developing treatment that allows us to capture the rich imagination that people with bipolar disorder have. We need to do work mapping out mental imagery in bipolar disorder (Holmes et al., 2011; Holmes, Geddes, Colom, \& Goodwin, 2008). We're also doing research on the 
effects of an online computer program, called OxIGen (Oxford Imagery Generation), on thinking style and mood in depression within a framework known as "cognitive bias modification". This builds on our earlier work of looking at the role of imagery of the future in depression (Lang, Blackwell, Hamer, Davison, \& Holmes, in press) and allows us to explore developing internet platforms for treatment delivery.

EJOP: Returning to the issue of interdisciplinarity and cross-fertilisation, EJOP is particularly keen to foster dialogues between different subdisciplines within psychology as well as between psychology and related sciences. In your work you have explored the links between experimental and clinical psychology, including psychotherapy. Traditionally the field of therapy has been informed by case studies conducted within the therapeutic practice. How are experimental procedures helpful for the work of the modern therapist?

Prof. Holmes: Case-studies are still very important and drive our early understanding of the phenomena we see in our clinical practice. We need case-studies, but we can also go further than that. In fact we have recently used case studies to test drive the application of some experimental work done in the lab (Blackwell \& Holmes, 2010). Psychology is a science, and in science you can do experiments. Experiments are the only way we know that we can test causal hypotheses, e.g. does Process $X$ influence Outcome Y? That's exactly what we can do in the lab, and that's why I think experimental psychopathology is a driver that can truly modernise and contribute to the development of therapies, alongside and informed by careful clinical work.

EJOP: Asking specifically about cognitive-behavioural therapy (CBT), a field you have been involved in most, what would you say are among the most important empirically-driven innovations in this area?

Prof. Holmes: It's difficult to single out a particular one, as there are so many fields that contribute, such as emotion, cognition or memory for example. It's using the body of science together in driving therapy that's the important thing to consider.

EJOP: Another important point of focus for EJOP is the link between theory, research and practice in psychology. Your work has successfully bridged the three and demonstrated how laboratory studies can shed light on what happens during the clinical treatment of patients. What are your thoughts on how this link can be further strengthened in psychology (or clinical psychology in particular) and what can be done to increase the applicability of psychological research? 
Prof. Holmes: We need to establish a European training platform for people in experimental psychopathology, to give them a really good experience of both clinical aspects as well as areas like brain-scanning, and then translating that knowledge and those skills back again. What I would really like to see is us all trying to encourage a new generation of researchers to gain experiences that they can effectively translate and bridge between disciplines. It may be from clinical psychology to brain-scanning but it could equally be taking a clinical issue and translating it to law. But what we need to do is help supervise and nurture people's talents and interests so they can spread across fields. There should be increased mobility and funding to allow people to bridge those skills and interests, particularly for students and early career researchers as they move into the next stage of their career. For early career stage researchers, making applied links and/or working between disciplines takes a lot of courage because their training can take longer and we should really seek to support and mentor them in this endeavour.

EJOP: Related to the above, psychologists also need to be able to communicate scientific results to broader audiences and everything that has to do with mental health in particular is scrutinised by laypeople and a variety of specialists from different fields. In this process researchers also become publicly accountable for their work. What do you think can be done by (clinical) psychologists to become more successful in disseminating their results?

Prof. Holmes: I think it is important to communicate to the public the results of one's science. When we've tried to do that in our lab, what's struck me and what's been really heartening is the range of responses that come back. Sometimes we have questions that come back from patients, but also we might have really interesting questions that come back, for example, from school children who've found descriptions of our studies and have then become interested in them as a science topic. It's great to see the interest that's been sparked off in them. So I think it's really about a spirit of curiosity about science in general; encouraging the next generation of people to do science is the really interesting question about communicating about science, whatever that science may be.

Within that, I do think that within mental health one has a responsibility to talk about it, to try and destigmatise it. Mental health problems will affect one in four people and it's very important that we put this information in the public domain positively, and handle it responsibly.

I am a fan of Open Access publishing - as that makes our scientific results available to a broad audience. 
On a practical note, if there are particular studies to communicate via the media to a broader audience, one way to help control the coverage is to have a very thoughtful and sensible press release which captures the important points. Perhaps in a sensitive field like this, that's always a good starting point, so that is carefully thought through and sensitively laid out - though ultimately one clearly cannot control everything that is written about one's work.

EJOP: Finally, and going back to another defining characteristic of EJOP, we are focused on promoting the work of graduate students and early career researchers, helping them communicate their research and become accustomed to the system of peer-review and academic publishing. You have been extremely successful in both developing a publication record and becoming established in your field of research. Your work has been awarded on different occasions by the British Psychological Society (the May Davidson Award, the Spearman Medal) and more recently you received the Comenius Early Career Psychologist Award from EFPA. What would be your advice for young and developing scholars on how to succeed in today's academic and professional world and make an impact from early on through their psychological research?

Prof. Holmes: Find a topic that you enjoy and niggles on your mind! Develop a base of mentors who care about you as a scientist and person as well as the science per se, and try and plan one step ahead. Develop a strong relationship with your supervisor but also get their advice and other people's advice about how to go to the next step. Plan ahead, but have integrity - do things because you really believe in them and you want to do them. I am an optimist - things will work out. Develop a good network of peers that can support you. For women in science or people who are in a minority in their particular field in whatever way, mentoring and peer support can be even more important. A good sense of humour is essential.

Get involved in contributing to your profession from the outside, like teaching and reviewing. It's about being an "academic citizen" - not just about focusing on your own narrow niche. And remember it's not always a straight line and that having lots of twists and turns is actually pretty interesting for you and your science!

\section{References}

Blackwell, S. E., \& Holmes, E. A. (2010). Modifying interpretation and imagination in clinical depression: a single case series using cognitive bias modification. Applied Cognitive Psychology, 24 (3), 338-350. doi: 10.1002/acp.1680 
Bonsall, M. B., Wallace-Hadrill, S. M. A., Geddes, J. R., Goodwin, G. M., \& Holmes, E. A. (2011). Nonlinear time-series approaches in characterizing mood stability and mood instability in bipolar disorder. Proceedings of the Royal Society B: Biological Sciences. doi: 10.1098/rspb.2011.1246

Hackmann, A., Bennett-Levy, J., \& Holmes, E. A. (2011). Oxford guide to imagery in cognitive therapy. Oxford: Oxford University Press.

Holmes, E. A. (2008). Memory, trauma and stress Guidelines on Memory and the Law: Recommendations from the Scientific Study of Human Memory. Leicester: British Psychological Society.

Holmes, E. A., Deeprose, C., Fairburn, C. G., Wallace-Hadrill, S. M. A., Bonsall, M. B., Geddes, J. R., et al. (2011). Mood stability versus mood instability in bipolar disorder: A possible role for emotional mental imagery. Behaviour Research and Therapy, 49(10), 707-713.

Holmes, E. A., Geddes, J. R., Colom, F., \& Goodwin, G. M. (2008). Mental imagery as an emotional amplifier: Application to bipolar disorder. Behaviour Research and Therapy, 46(12), 1251-1258.

Holmes, E. A., James, E. L., Kilford, E. J., \& Deeprose, C. (2010). Key steps in developing a cognitive vaccine against traumatic flashbacks: visuospatial Tetris versus verbal Pub Quiz. PLoS ONE, 5(11), e13706. doi: doi:10.1371/journal.pone.0013706

Holmes, E. A., \& Mathews, A. (2010). Mental imagery in emotion and emotional disorders. Clinical Psychology Review, 30(3), 349-362. doi: doi: 10.1016/j.cpr.2010.01.001

Holmes, E. A., Sandberg, A., \& Iyadurai, L. (2010). Erasing trauma memories: Is this what the science suggests or even what we want to do? [Letter to the editor]. British Journal of Psychiatry Online, 190(1), 81 a.

Lang, T. J., Blackwell, S. E., Hamer, C. J., Davison, P., \& Holmes, E. A. (in press). Cognitive bias modification using mental imagery for depression: developing a novel computerized intervention. European Journal of Personality.

National Institute for Health and Clinical Excellence. (2005). Post-traumatic stress disorder (PTSD): the management of PTSD in adults and children in primary and secondary care. London National Institute for Health and Clinical Excellence.

Pictet, A., Coughtrey, A. E., Mathews, A., \& Holmes, E. A. (2011). Fishing for happiness: The effects of positive imagery on interpretation bias and a behavioral task. Behaviour Research and Therapy. doi: doi 10.1016/j.brat.2011.10.003 